\title{
Ewingella americana : une bactérie pathogène émergente isolée de selles diarrhéiques à l'Hôpital de l'Amitié Sino-Guinéenne de Kipé/Conakry
}

\section{Ewingella americana: an emerging bacterial pathogen from diarrhea at China and Guinea friendship hospital of Kipé/Conakry}

\author{
Makanéra $\mathrm{A}^{1,2^{*}}$, Condé $\mathrm{M}^{2}$, Diallo $\mathrm{MA}^{2}$, Sy $\mathrm{O}^{2}$, Diakité $\mathrm{T}^{2}$, Condé $\mathrm{M}^{2}$, Barry $\mathrm{AO}^{2}$, Camara $\mathrm{D}^{2}$
}

Laboratoire biomédical de l'Hôpital de l'Amitié Sino-Guinéenne, Kipé, Conakry, Guinée

2- Université Gamal Abdel Nasser de Conakry, Faculté de Sciences et Techniques de la Santé, Département des Sciences fondamentales ; Conakry, Guinée

\begin{abstract}
*Auteur pour Correspondances: Abdoulaye MAKANERA, Laboratoire biomédical de l'Hôpital de l'Amitié Sino-Guinéenne, Kipé, Cité des Médecins, Commune Ratoma 030 BP : 710 Université Gamal Abdel Nasser Conakry ; FSTS Département des Sciences fondamentales ; Conakry (Guinée); e-mail: abdmak@ yahoo.fr RESUME :
\end{abstract}

Ewingella americana (E. americana), est une bactérie appartenant à la famille des Enterobacteriaceae autrefois rarement associée à des infections humaines. L'objectif était de décrire une souche d'E. americana isolée de selles diarrhéiques à l'Hôpital de l'Amitié Sino-Guinéenne de Kipé/Conakry. L'échantillon de selles diarrhéiques a été prélevé chez un patient de sexe masculin, âgé de 18 ans. La culture a été faite sur différents milieux gélosés pendant 24 heures à $37^{\circ} \mathrm{C}$. L'identification bactérienne, l'antibiogramme et la détermination des concentrations minimales inhibitrices ont été faits sur l'automate Vitek2 Compact 15. La souche d'E. Americana identifiée était sensible à la majorité des antibiotiques testés. En revanche, cette souche était résistante à la céfoxitine, l'amikacine, et l'acide nalidixique et présentait une sensibilité intermédiaire à la céfalotine, la gentamicine et la tobramycine. Ce présent travail rapporte à notre connaissance, la première description d' $E$. americana isolée des selles diarrhéiques avec une multi-résistance à certains antibiotiques.

Mots clés : Ewingella americana, diarrhées, antibiotiques, sensibilité.

\begin{abstract}
ABSRACT
Ewingella americana (E. americana) is the only species of the genus of Ewingella in the familly Enterobacteriaceae. It is becoming reported in various human infections. The aim of this paper was to describe E. americana strain isolated from human diarrhea at China and Guinea friendship hospital of Kipé/Conakry. A sample of diarrheal stools from 18 -Years old patient was studied. After 24 hours coprocultures at $37^{\circ} \mathrm{C}$, the bacterial identification and antibiogram were processed on Vitek 2 Compact 15 (Biomérieux, Marcy l'Etoile, France). The bacterial isolate was identified as E. americana. This isolate was susceptible to majority (12/18) of antibiotics tested, but it was resistant to cefoxitin, amikacin and nalidixic acid, and showed intermediate sensitivity to cefalothin, gentamicin and tobramycin. We report here, to our knowledge the first case of $E$. americana isolated from human diarrhea, with multi-drug resistance.
\end{abstract}

Key words: Ewingella americana, diarrhea, antibiotics, sensitivity.

\section{Introduction}

Ewingella americana est actuellement l'unique espèce du genre Ewingella appartenant à la famille des Enterobacteriaceae. Cette bactérie a été décrite pour la première fois en 1983 à partir d'échantillon clinique comme un nouveau groupe de bactérie appartenant à la famille des Enterobacteriaceae [14]. E. americana est une bactérie rare, préalablement connue sous l'appellation de groupe entérique pathogène 40 [1]. Elle présente les caractéristiques ci-après : Il s'agit de bacilles à Gram négatif, oxydase négative, catalase positive, anaérobie facultative, indole négatif et fermentant le lactose. E. americana est négative pour le saccharose, l'arabinose et l'utilisation de malonate [1-4].Ewingella americana est rarement associée à des infections chez l'Homme. Cependant, quelques cas d'infections à Ewingella americana ont été écrits. En effet, certaines souches de cette bactérie avaient été isolées de péritonites, d'expectorations, de sang par hémocultures, de conjonctivites, de pus d'ostéomyélites et d'arthrites septiques [1-6].
Certains auteurs ont rapporté l'isolement d'Ewingella americana à partir d'intestins de mollusques et de limaces, de champignons, de viande, de carcasses fraiches d'animaux $[1,6]$.

L'objectif de ce présent travail était de décrire pour la première fois, à notre connaissance, une souche d'Ewingella americana isolée de selles diarrhéiques à l'Hôpital de l'Amitié Sino-Guinéenne de Kipé/Conakry.

Description du cas

Un échantillon de selles diarrhéiques a été prélevé le 19 novembre 2018 chez un patient en provenance du service des maladies infectieuses et tropicales de l'Hôpital national Donka. Le patient de sexe masculin était âgé de 18 ans. Il faisait des selles diarrhéiques de couleur verdâtre, avec absence d'hématies. Des épisodes de vomissements ont été enregistrés. Le patient était fébrile avec une hyperthermie de $39^{\circ} \mathrm{C}$. L'échantillon soumis à un examen parasitologique direct à l'état frais au microscope optique (Microscope XS-213, China) était négatif. Le diagnostic du paludisme par la 
méthode de goutte épaisse était également négatif. Les coprocultures ont été réalisées sur différents milieux gélosés: gélose MacConkey, gélose Salmonella-Shigella, gélose Columbia au sang de mouton 5\%, gélose CLED, gélose nutritive. L'incubation a été faite à l'étuve GRP 9080 (Sumsung Laboratory Instrument Co., Ltd, Shanghai, China) pendant 24 heures à $37^{\circ} \mathrm{C}$. Des colonies bactériennes uniformes isolées des primocultures ont été étalées sur lames porte-objet, séchées et colorées par la méthode de Gram afin de vérifier leur pureté, étape clé précédant les analyses à l'automate Vitek 2 Compact 15 (Biomérieux, Marcy l'Etoile, France). Des subcultures avaient également été effectuées afin de tester la reproductibilité des résultats. Toutes les colorations par la méthode de Gram des colonies bactériennes issues des primocultures et des subcultures ont révélé la présence des bacilles à Gram négatif. L'identification bactérienne, les antibiogrammes et la détermination des concentrations minimales inhibitrices (CMI) ont été réalisés à l'aide de l'automate Vitek 2 compact 15. Les cartes Vitek 2 GN utilisant 64 réactions différentes pour l'identification bactérienne ont montré que la seule et unique souche bactérienne isolée des primocultures et subcultures des selles diarrhéiques appartenait à l'espèce Ewingella americana. L'antibiogramme et la détermination des CMI ont été réalisés à l'aide des cartes Vitek 2 AST-N 233. Ces cartes prêtes à l'emploi et contenant 18 antibiotiques et associations d'antibiotiques appartenant à différentes familles (Tableau I) sont destinés aux bactéries à Gram négatif fermentaires et non fermentaires. Les antibiotiques non requis en fonction des germes identifiés sont éliminés automatiquement par le système Vitek 2 compact 15. Les critères et normes d'interprétation de Clinical and Laboratory Standards Institute (CLSI) ont été appliqués pour l'interprétation des résultats de l'antibiogramme et la détermination des CMI. Le logiciel Advanced Expert System (AES) compare les CMI de l'instrument et l'identité du germe identifié aux phénotypes standard de ce germe (CLSI + Natural Resistance).L'AES communique les résultats obtenus sous forme de rapports récapitulatifs et de propositions. Les résultats de l'antibiogramme et des CMI des différents antibiotiques testés sont présentés dans le Tableau I. Les données de l'antibiogramme ont montré que pour les bêta-lactamines, la souche d'E. americana isolée de selles diarrhéiques était sensible à l'ampicilline, la ticarcilline, le céfotaxime, la ceftazidime, l'ertapenème, l'imipenème, les associations pipéracilline/tazobactam et amoxicilline/acide clavulanique, avec valeurs de CMI faibles (Tableau I). Cette souche était par contre résistante à la céfoxitine avec une CMI élevée (CMI $\geq 64 \mu \mathrm{g} / \mathrm{ml})$ et présentait une sensibilité intermédiaire à la céfalotine $(\mathrm{CMI}=16$ $\mu \mathrm{g} / \mathrm{ml})$. D'autre part, les résultats de l'antibiogramme concernant les aminosides ont révélé la résistance de la souche d'E. americana étudiée à l'amikacine avec une CMI élevée (CMI $\geq$ $64 \mu \mathrm{g} / \mathrm{ml})$. Cependant, cette souche présentait une sensibilité intermédiaire à la gentamicine $(\mathrm{CMI}=8$ $\mu \mathrm{g} / \mathrm{ml})$ et à la tobramycine $(\mathrm{CMI}=8 \mu \mathrm{g} / \mathrm{ml})$. Par ailleurs, les résultats de l'antibiogramme pour les quinolones ont montré que la souche d'E. americana étudiée était sensible à la ciprofloxacine et à l'ofloxacine avec des CMI faibles (Tableau I). En revanche, cette souche était résistante à l'acide nalidixique avec une CMI élevée (CMI $\geq 32 \mu \mathrm{g} / \mathrm{ml})$. Enfin, l'étude de la sensibilité aux sulfamides et aux nitrofurantoines de la souche d'E. americana étudiée a révélé que cette souche était sensible d'une part à l'association triméthoprime/sulfaméthoxazole et d'autre part à la nitrofurantoine (Tableau I).

\section{$\underline{\text { Discussion }}$}

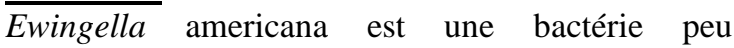
fréquemment associée à des infections humaines. Cependant, les infections humaines à Ewingella americana sont de plus en plus décrites à travers le monde [1-5]. Malgré que cette bactérie soit peu connue dans la littérature comme les autres entérobactéries, son caractère pathogène n'est plus en doute. En effet, différents cas d'infection à $E$. americana décrits dans la littérature et rapportés dans différents pays étaient associés à des bactériémies, des expectorations, des conjonctivites, de pus, de liquide péritonéal, d'urines, de selles, de pus d'ostéomyélites et d'arthrites septiques [16].Dans ce présent travail, le seul et unique germe isolé dans l'échantillon de selles diarrhéiques était E. americana et la recherche d'éventuels parasites intestinaux était négative. Ceci pourrait suggérer le caractère pathogène d'E. americana qui n'est pas habituellement connue dans la flore normale du tube digestif. Farmer et al., cités par certains auteurs [3,4] avait déjà rapporté deux cas d'isolement d'E. americana de selles non diarrhéiques, suggérant ainsi que ce présent travail pourrait être à notre connaissance, la première description d'isolement d'E. americana des selles diarrhéiques.

\section{Caractère d'émergence d'Ewingella americana}

De nombreux cas d'isolement d'Ewingella americana chez l'Homme ont été rapportés ces deux dernières décennies. Ainsi, l'incidence des infections à Ewingella americana semble avoir augmenté ces deux dernières décennies à travers le monde. Cette tendance à l'augmentation pourrait continuer, entre autres raisons, sous la pression de sélection exercée par l'usage abusif et des pescriptions anarchiques des antibiotiques. Ce constat semble en rapport avec la définition des maladies infectieuses émergentes de Centers for Diseases Control (CDC) and Prevention d'Atlanta (USA) selon laquelle : «Les maladies infectieuses 
émergentes sont des maladies infectieuses dont l'incidence chez l'Homme a augmenté au cours des deux dernières décennies ou qui menace d'augmenter dans un avenir proche » [7]. L'acquisition d'un phénotype de multi-résistance pourrait être un facteur important d'évolution de l'écologie bactérienne. Woolhouse et GowtageSequeria en 2005, dans leur étude sur les mécanismes associés à l'émergence ou à la réémergence des germes pathogènes infectant l'Homme, ont établi une classification de ces mécanismes en dix catégories rapportées en 2018 par Makanéra et al., [7]. L'ordre de classification est fonction de l'importance du nombre d'espèces pathogènes associées à ces mécanismes. L'évolution des pathogènes due à la résistance aux antimicrobiens ou à l'augmentation de leur virulence occupe le $5^{\text {ème }}$ rang de cette classification. Variabilité des profils de sensibilité aux antibiotiques

L'étude de la sensibilité aux bêta-lactamines de la souche d'E. americana a montré que nos résultats sont en partie semblables à ceux trouvés par Ryoo et al. [3] d'une part, qui ont rapporté la sensibilité de leur souche d'E. americana à l'ampicilline, l'imipenème, la pipéracilline, la pipéracilline/tazobactam, l'ampicilline/sulbactam, [3], et d'autre part, à ceux de Kati et al., rapportés par Ryo et al., [3], qui ont trouvé une sensibilité de leur souche à l'ampicilline, le céfépime et céfotaxime [7]. En revanche contrairement aux résultats de ce présent travail, Ryoo et al., [3] avaient trouvé une sensibilité de leur souche à la céfoxitine, mais une résistance à la céfalotine. D'autre part, nos résultats sont très différents de ceux trouvés par certains auteurs qui ont rapporté la résistance de leurs souches d'E. americana aux céphalosporines de troisième génération en l'occurrence la ceftriaxone et la ceftazidime $[8,9]$. De même, nos résultats sont différents de ceux trouvés en 2014 par Li et al., [10] qui ont décrit une souche d'E. americana résistante à l'ampicilline $(\mathrm{CMI} \geq 32 \mu \mathrm{g} / \mathrm{ml})$, et à l'ertapenème $(\mathrm{CMI} \geq 8 \mu \mathrm{g} / \mathrm{ml})$. Cette souche était par contre sensible à la ceftazidime et à l'imipénème et avait une sensibilité intermédiaire à la ceftriaxone (CMI= $\mu \mathrm{g} / \mathrm{ml})$ [10]. Par ailleurs, nos résultats sont en partie différents de ceux trouvés en 2007 par Pound et al., [2] qui ont décrit une souche d'E. americana, multirésistante aux antibiotiques dont l'ampicilline, la céfoxitine, l'ertapénème et l'imipenème. Cependant, cette souche présentait une sensibilité intermédiaire au céfotaxime, à la ceftazidime, et à la pipéracilline/tazobactam. Ces résultats montrent ainsi une diversité des profils de sensibilité des souches d'E. americana aux bêta-lactamines. Pour les aminosides les résultats de l'antibiogramme étaient en général différents de ceux précédemment trouvés par certains auteurs. En effet, les résultats rapportés par Ryoo et al., [3] ont montré une sensibilité totale de leurs souches d'E. americana à l'amikacine et à la gentamicine. Cependant, ces auteurs n'avaient pas testé la sensibilité de leurs souches à la tobramycine comme dans la présente étude [6]. De même, les données rapportées par Hassan et al., [1] qui ont décrit une sensibilité de leur souche à l'amikacine sont différentes des nôtres [1]. Cependant, ces auteurs n'ont pas testé la sensibilité de leur souche isolée du liquide synovial à d'autres aminosides. D'autre part, nos résultats sont également différents de ceux rapportés par Yazici et al., [8] ont décrit une souche d'E. americana sensible à la gentamicine, la tobramycine, l'amikacine, [8]. En revanche, nos résultats sont proches de ceux de Bukhari et al., [9] qui ont rapporté dans leur étude une résistance franche de leur souche d'E. americana à l'amikacine (CMI>32 $\mu \mathrm{g} / \mathrm{ml})$, la gentamicine $(\mathrm{CMI}>8 \mu \mathrm{g} / \mathrm{ml})$ et la tobramycine $(\mathrm{CMI}>8 \mu \mathrm{g} / \mathrm{ml})$ [9]. En ce qui concerne ces deux derniers antibiotiques (gentamicine et tobramycine), notre souche d'E. americana présentait une sensibilité diminuée (ou sensibilité intermédiare), suggérant ainsi une différence par rapport aux données rapportées par ces auteurs [8,9]. Ces données indiquent ainsi la variabilité de la sensibilité d' $E$. americana aux aminosides. La sensibilité de la souche d'E. americana aux quinolones montre que nos résultats sont semblables à ceux trouvés par d'autres auteurs qui ont rapporté la sensibilité de leurs souches d'E. americana à la ciprofloxacine et à l'ofloxacine [3]. Cependant, ces auteurs n'avaient pas testé la sensibilité de leurs souches à l'acide nalidixique. En revanche, nos résultats sont différents de ceux rapportés par Pound et al., [2] qui ont montré une résistance de leur souche à la ciprofloxacine [2]. Ces différentes données montrent que les quinolones pourraient être considérées comme étant des antibiotiques généralement actifs sur E. americana. Cependant, cette bactérie est bien capable d'acquérir des phénotypes de résistance à ces antibiotiques. Les résultats de tests de sensibilité aux sulfamides de la souche étudiée dans ce présent travail sont semblables à ceux trouvés dans la plupart des études antérieures [2,6] qui ont montré la sensibilité de leur souche d'E. americana à cette association de sulfamides. En revanche, nos résultats sont différents de ceux trouvés en 2008 par Bukhari et al., [9] qui ont décrit une résistance de leur souche à ces antibiotiques. Ainsi, les données rapportées sur la sensibilité aux sulfamides d'E. americana par différents auteurs varie d'une souche à l'autre. A ce jour, il n'existe pas à notre connaissance de données antérieures sur la sensibilité d'E. americana à la nitrofurantoine. En effet, aucun des auteurs mentionnés ci-dessus n'avait testé la sensibilité de leurs souches d'E. americana à la nitrofurantoine. Ainsi les résultats de ce présent travail pourraient 
constituer la première description de la sensibilité d'E. americana à la nitrofurantoine.

Conclusion: Ce présent travail montre qu'E. americana est une bactérie pathogène émergente pouvant être associée à des cas de diarrhées chez l'Homme. Cette bactérie est généralement sensible aux antibiotiques. Cependant, le profil de sensibilité aux antibiotiques peut varier d'une souche à l'autre. Ainsi, l'acquisition de phénotypes de multirésistance aux antibiotiques pourrait conduire à la propagation de cette espèce chez l'Homme, l'Animal et dans l'environnement.

\section{Conflit d'intérêt : Aucun.}

Aspect éthique : Le consentement éclairé du patient a été obtenu pour cette étude.

Remerciements: Nous remercions le Ministère de la Santé de la République de Guinée et la République populaire de Chine à la réalisation de ce travail.

\section{REFERENCES:}

1. Hassan S, Amer S, Mittal C, Sharma R. Ewingella Americana: An Emerging True Pathogen. Case Reports in Infectious Diseases, 2012, Article ID 730720, 2 pages doi:10.1155/2012/730720

2. Pound MW, Tart SB, and Okoye O. MultidrugResistant Ewingella Americana: A Case Report and Review of the Literature. The Annals of Pharmacotherapy. 2007; 41: 2066-2070

3. Ryoo NH, Ha JS, Jeon DS, Kim JR, Kim HC. A case of pneumonia caused by Ewingella americana in a patient with chronic renal failure. J Korean Med Sci., 2005; 20:143-5.
4. Maertens J, Delforge $M$, Vandenberghe $P$, Boogaerts M, Verhaegen J. Catheter-related bacteremia due to Ewingella americana. Clin Microbiol Infect., 2001; 7:103-4. DOI 10.1016/j.clinmicnews.2004.11.004

5. Da Costa PS, Tostes MM, de Carvalho Valle LM, "A case of keratoconjunctivitis due to Ewingella Americana and a review of unusual organisms causing external eye infections," The Brazilian Journal of Infectious Diseases, 2000; 4(5) : 262267.

6. Lyon WJ and Milliet JB, "Microbial flora associated with Louisiana processed frozen and fresh nutria (Myocastor coypus) carcasses," Journal of Food Science, 2000 ; 65 (6)1041-1045.

7. Makanéra A, Condé M, Diallo MA, Condé M, Camara D. Sphingomonas paucimobilis: Un phénotype de multi-résistance inhabituel chez un probable pathogène émergent. Rev Mali Infect Microbiol, 2018; (12) : 17-24

8. Yazici E, Birbir M, Caglayan P. ICAMS 20187thInternational Conference on Advanced Materials and Systems https. https://doi.org/10.24264/icams2018.VI.15

9. Bukhari SZ, Husain WM, Fatani MI, Ashshi AM. Multidrug-resistant Ewingella americana. Saudi Med J, 2008 ; 29(7) :1051-1053

10. Li L, Shen J, Tao J, Xue Z. Peritonitis caused by Ewingella americana in a patient with peritoneal dialysis: a case report, Journal of Medical Case Reports 2014, 8:86

http://www.jmedicalcasereports.com/content/8/1/86

Tableau I: Sensibilité aux antibiotiques de la souche d'E. americana et les Concentrations minimales inhibitrices

\begin{tabular}{lc}
\hline Antibiotiques & Sensibilité $(\mathbf{C M I}=\boldsymbol{\mu} \mathbf{g} / \mathbf{m l} \mathbf{~}$ \\
\hline Ampicilline & $\mathrm{S}(\leq 2)$ \\
Céfalotine & $\mathrm{I}(=16)$ \\
Ticarcilline & $\mathrm{S}(\leq 8)$ \\
Nitrofurantoine & $\mathrm{S}(\leq 16)$ \\
Pipéracilline/Tazobactam & $\mathrm{S}(\leq 4)$ \\
Amoxicilline/acide clavulanique & $\mathrm{S}(\leq 2)$ \\
Cefoxitine & $\mathrm{R}(\geq 64)$ \\
Cefotaxime & $\mathrm{S}(\leq 1)$ \\
Ceftazidime & $\mathrm{S}(=8)$ \\
Ertapenème & $\mathrm{S}(=2)$ \\
Imipenème & $\mathrm{S}(\leq 0,25)$ \\
Amikacine & $\mathrm{R}(\geq 64)$ \\
Gentamicine & $\mathrm{I}(=8)$ \\
Tobramycine & $\mathrm{I}(=8)$ \\
Acide nalidxique & $\mathrm{R}(\geq 32)$ \\
Ciprofloxacine & $\mathrm{S}(=1)$ \\
Ofloxacine & $\mathrm{S}(=2)$ \\
Triméthoprime/sulfaméthoxazole & $\mathrm{S}(\leq 20)$ \\
\hline
\end{tabular}

S: sensibilité, $R$ : résistance, I: intermédiaire 